Esta revista forma parte del acervo de la Biblioteca Jurídica Virtual del Instituto de Investigaciones Jurídicas de la UNAM

\title{
El nuevo modelo de gobernanza electoral: un cambio desatinado
}

\author{
The New Model of Electoral Governance: \\ Another Mistaken Change
}

\section{Karolina M. Gilas*}

\section{Sumario:}

I. Introducción.

II. Gobernanza electoral, conceptos y categorías de análisis.

III. Evolución de la gobernanza electoral en México.

IV. Nuevo modelo de gobernanza.

V. ¿Un modelo mejor?

VI. Conclusiones.

* Profesora en la Facultad de Ciencias Políticas y Sociales de la UNAM e investigadora en el Centro de Capacitación Judicial Electoral del Tribunal Electoral del Poder Judicial de la Federación; miembro del Sistema Nacional de Investigadores. 
Esta revista forma parte del acervo de la Biblioteca Jurídica Virtual del Instituto de Investigaciones Jurídicas de la UNAM

\section{Resumen:}

La reforma electoral de 2014 modificó profundamente el sistema electoral mexicano, al establecer un nuevo modelo de gobernanza electoral. México pasó de un sistema dual (federal y local), a tener un modelo altamente centralizado en términos de la legislación y de competencias de las autoridades. El presente artículo analiza los cambios introducidos a partir de las categorías propias de la gobernanza electoral, mediante la comparación del modelo actual y el anterior. La conclusión es que las modificaciones introducidas no logran establecer un modelo viable, y que las problemáticas importantes, que desde hace años afectaban al sistema electoral mexicano, no fueron eliminadas por la reforma (complejidad, lagunas legales, judicialización, etcétera). Por lo tanto, es altamente probable que el ciclo de la gobernanza siga su camino, lo que llevará en un futuro próximo a otra reforma electoral.

\section{Abstract:}

The 2014 electoral reform profoundly changed the Mexican electoral system, establishing a new model of electoral governance. Mexico went from a dual (federal and local) system to have a highly centralized model, both in terms of legislation and jurisdiction. The article analyzes the changes from the very categories of electoral governance, comparing the current and the previous models. The conclusion is that the amendments fail to establish a viable model and that some important issues that for years affected Mexican electoral system (complexity, legislative gaps, juridification), were not eliminated by the reform. Therefore, it is highly likely that the cycle of governance will continue, and that in the near future we may expect another electoral reform.

Palabras clave: gobernanza electoral, sistema electoral mexicano, autoridades electorales.

Keywords: electoral governance, Mexican electoral system, electoral authorities. 


\section{Introducción}

La organización de las elecciones, por mucho tiempo, no fue un campo relevante en el estudio de las ciencias políticas y del derecho. Las democracias consolidadas, al estar satisfechas con sus procesos electorales, consideraban ese tema como zanjado. Fueron las transiciones de la tercera ola las que llamaron la atención sobre la problemática electoral, relativa a la administración electoral y a las reglas de competencia.

Muchos de los países que transitaron a la democracia dentro de la tercera ola solían tener regímenes autoritarios o dictatoriales, pero celebraban elecciones periódicas, aunque no libres ni competitivas. Elecciones operadas por los gobiernos autoritarios producían resultados a la medida, lo que aseguraba el dominio del mismo gobierno. Su finalidad podía ser diferente en los distintos momentos de vida del régimen, pero, en general, permitían canalizar las pugnas internas, servían como mecanismos de participación reglamentada de los partidos satélite o de oposición y, con ello, ratificaban la legitimación del sistema en el ámbito doméstico e internacional. ${ }^{1} \mathrm{Al}$ ser la administración pública la responsable de la organización de las elecciones fraudulentas durante la existencia de los regímenes autoritarios, el diagnóstico que acompañó los procesos de transición determinó que era necesario "sacar las elecciones de manos del gobierno", por lo que una parte importante de los procesos de liberalización y transición de estos países consistió en desmantelar esas maquinarias de organización y control electoral, para crear nuevas instituciones, autónomas e independientes del gobierno, especializadas en la función electoral, capaces de dotar de confianza a los procesos electorales.

A su vez, la dinámica propia de las nuevas democracias - un alto grado de desconfianza en la autoridad y en los actores políticos, existencia de partidos herederos del sistema y una alta conflictividad electoralconllevó también la necesidad de establecer el control judicial de los resultados electorales y de las decisiones de las autoridades en la materia. De ahí que muchos países han introducido mecanismos de revisión de los resultados y conflictos electorales en la sede jurisdiccional, mediante la creación de tribunales electorales, u otorgando esa facultad a un tribunal constitucional o administrativo existente.

La (re)aparición de la temática electoral como objeto de estudio, en una dinámica nueva que implicó análisis de los diseños institucionales y reglas de juego, adicionalmente a los temas tradicionales de sistemas

\footnotetext{
1 Nohlen, Dieter, Sistemas electorales y partidos políticos, México, UNAM-FCE, 1994.
} 
electorales, requirió de construcción de un nuevo concepto que permitiera abordar la complejidad de los procesos. Una de esas propuestas, y la más sólida, es la de gobernanza electoral.

La gobernanza electoral reaparece periódicamente en México, con mayor fuerza, como objeto de estudio. Nuestro ciclo de reformas requiere de análisis, las nuevas reglas del juego deben ponerse a escrutinio por la práctica y la academia. Desde 2014 estamos, otra vez, frente a un nuevo ciclo. La reforma impactó el sistema electoral mexicano de manera profunda, pues dio pie a la creación de un nuevo modelo de gobernanza electoral.

En el presente trabajo se revisará el modelo de gobernanza electoral en México a partir de la reforma de 2014. El artículo parte de un estudio de gobernanza electoral, y establece las categorías de análisis, para aplicarlas al análisis del modelo anterior y de los cambios introducidos por la última reforma. Finalmente, utiliza las mismas categorías para analizar el desempeño del nuevo modelo de gobernanza en los procesos electorales de 2015, y establece conclusiones acerca de la viabilidad del mismo.

\section{Gobernanza electoral, conceptos y categorías de análisis}

El concepto de la gobernanza electoral, como marco de análisis de las elecciones, apareció en la ciencia política en la época de la tercera ola de democratización, cuando las dinámicas de las democracias nacientes colocaron en el centro de atención al diseño y administración de los procesos electorales. ${ }^{2}$ Otro factor que explica el renovado interés en los asuntos electorales fue el gran número de problemáticas en el desarrollo de los procesos electorales en las democracias consolidadas, que visibilizaron las deficiencias en la funcionalidad del diseño electoral. Un ejemplo ya clásico son las elecciones presidenciales de 2000 en Estados Unidos, donde hubo problemas con el diseño de las boletas y con el uso de las máquinas para votar, entre otros. ${ }^{3}$ Otras democracias establecidas tampoco resultaron estar exentas de importantes fallas en sus sistemas electorales, que frecuentemente son resultado de errores en la actuación de las autoridades electorales; ${ }^{4}$ por ejemplo, en el mismo trabajo, James

2 Marchetti, Vitor, "Governança Eleitoral: O Modelo Brasileiro de Justiça Eleitoral”, DADOS-Revista de Ciências Sociais, vol. 51, núm. 4, 2008, pp. 865-893.

3 Mozaffar, Shaheen y Schedler, Andreas, "The Comparative Study of Electoral Governance”, International Political Science Review, vol. 23, enero de 2002, pp. 5-27.

4 James, Toby, ponencia "Centralizing Electoral Management: Lessons from the U. K., en Congress of the American Political Science Association, agosto de 2013, p. 2; 
señala otros ejemplos de una organización electoral deficiente: largas filas en las elecciones británicas de 2010 que impidieron a muchos electores emitir su voto; mal diseño de las boletas en la elección de 2007 en Escocia; retraso en el conteo de votos a causa de problemas técnicos y de operación en las elecciones de 2013 en Kenia; o doble voto en las elecciones 2013 en Malasia, donde los funcionarios electorales no aplicaron correctamente el líquido indeleble. ${ }^{5}$ Finalmente, se estima que los funcionarios electorales en Canadá, en 2011, cometieron durante la jornada electoral, en promedio, más de 500 errores administrativos graves en cada distrito.

La gobernanza electoral, entendida como "el conjunto extenso de actividades que crea y mantiene el marco institucional en el cual se desarrollan los comicios y la competencia electoral", abarca tanto la elaboración de reglas, como su aplicación y adjudicación. ${ }^{6}$ Trabajos posteriores de Medina y Ramírez ${ }^{7}$ añaden a esto un elemento más: revisión regional por parte de los órganos internacionales de derechos humanos, a partir del creciente desarrollo del control internacional de las legislaciones nacionales. Desde esta modificación, se crea la definición más completa de gobernanza:

En resumen, la gobernanza electoral es la relación que se presenta entre los actores políticos con las autoridades electorales en el marco de un proceso comicial competitivo con reglas conocidas y procedimientos adoptados por los participantes, siendo las decisiones legislativas con las que inicia el ciclo, el cual se termina con una resolución jurisdiccional o con una recomendación de modificaciones legislativas [por parte del órgano nacional o regional]. ${ }^{8}$

De esa definición se desprenden claramente cuatro etapas que, en conjunto, son el ciclo de gobernanza electoral: diseño de reglas, aplicación de reglas, resolución de disputas y revisión regional.

disponible en https://sites.google.com/site/electoralintegrityproject4/events-1/chicago-28aug-2013 (fecha de consulta: 19 de marzo de 2016).

5 Idem.

6 Mozaffar, Shaheen y Schedler, Andreas, op. cit.

7 Medina Torres, Luis Eduardo y Ramírez Díaz, Edwin Cuitláhuac, "Electoral Governance: More Than Just Electoral Administration”, Mexican Law Review, vol. VIII, núm. 1, 2015, pp. 33-46.

8 Medina, Luis Eduardo, ponencia "La gobernanza electoral mexicana y las reformas en el periodo de 1977 a 2014", en el XXV Congreso Nacional y V Internacional de Estudios Electorales, San José, Costa Rica, noviembre de 2014. 
La primera etapa se refiere al proceso legislativo mediante el cual los actores involucrados crean y adoptan las reglas que van a guiar la organización de las elecciones, las reglas de participación, de resolución de conflictos y de validez de los resultados. En la segunda etapa, la de aplicación, las autoridades y demás actores que participan en los procesos comiciales (candidatos, partidos, medios de comunicación, organizaciones ciudadanas, etcétera), ponen en práctica las reglas previamente diseñadas. Después, en la tercera etapa (ésta puede ser parcialmente coincidente con la de aplicación), los órganos jurisdiccionales resuelven los conflictos que se presentaron entre las autoridades y otros actores involucrados, relativos a los resultados de los comicios o a la aplicación de las reglas de juego. Con ello, los tribunales realizan la interpretación de las reglas e, incluso, pueden modificarlas, invalidarlas o crear reglas nuevas. Las decisiones de los órganos jurisdiccionales son susceptibles de control de convencionalidad en la cuarta etapa. Al ejercer ese control, los órganos internacionales pueden imponer a las autoridades nacionales cierta interpretación, inaplicación, creación o modificación de las reglas del juego electoral. El ciclo puede terminar en la etapa tres, de adjudicación de conflictos, o en la cuatro, de revisión regional. Como consecuencia de las interpretaciones realizadas y experiencia adquirida, se puede llegar a una modificación de las reglas realizada por el Legislativo, una reforma electoral, con la que el ciclo vuelve a iniciar.

La corriente parte de la premisa de que un estudio exhaustivo de los regímenes electorales no puede quedarse únicamente en el análisis de las reglas, sino que debe tomar bajo escrutinio a las instituciones que las aplican, el grado de su cumplimiento y su impacto en la actuación de otros actores políticos. Al ser un concepto transversal, la gobernanza electoral abarca el estudio de todos los procesos relacionados con la organización de los procesos electorales, como son el diseño de las autoridades electorales, integración de congresos electorales, invalidez de los comicios, regulación de propaganda electoral y regulación de financiamiento de partidos. De esa manera, el concepto permite abordar la complejidad de los procesos electorales, partiendo de la creación de las reglas que regulan la participación de los actores políticos, ciudadanos y autoridades, la organización del proceso y la resolución de conflictos, y reflejando el ciclo de gobernanza: el quién, cómo, cuándo y dónde del proceso electoral.

Esta descripción nos lleva a la conclusión de que la gobernanza electoral es de naturaleza cíclica, y que los procesos que la conforman pueden sufrir modificaciones desde una nueva reforma electoral, como a partir 
de la interpretación jurisdiccional. Como señalan Medina y Ramírez, " "la gobernanza electoral es un ciclo que inicia en el diseño legislativo, pasa a través de la administración y justicia electoral interna, con la posibilidad de terminar en la revisión en un sistema regional de protección de derechos humanos". A partir de ello, los mismos autores postulan análisis de la gobernanza electoral a partir de las siguientes categorías:

1) Sistema de reglas: regional, nacional o subnacional; describe el número y tipo de legislaciones aplicables, así como relación entre ellas.

2) Niveles de administración: nacional o subnacional; se refiere a los órganos de gobierno responsables de la tarea electoral, y a la relación entre ellos.

3) Órganos electorales: administrativos, jurisdiccionales, de revisión regional; analiza las autoridades involucradas en la tarea de organización electoral, su tipo y ámbito de competencia.

4) Actores políticos: ciudadanos, candidatos y partidos; se refiere a la participación electoral de los distintos actores políticos, reflejando la existencia y la regulación de la competencia electoral. ${ }^{10}$

Las categorías señaladas nos permiten analizar los cambios en el modelo mexicano de gobernanza electoral y evaluar su efectividad en el proceso electoral de 2015.

\section{Evolución de la gobernanza electoral en México}

México es un ejemplo de los modelos de gobernanza electoral basados en la creación de autoridades autónomas y altamente especializadas en la materia, que derivan de las particularidades de los procesos de liberalización del régimen autoritario y, después, de la transición a la democracia. Ambos procesos transitaron, básicamente, por la ruta electoral.

Existe ya literatura abundante sobre el tema y las dinámicas que, a partir de la elección presidencial de 1976 en la que fue registrado solamente un candidato, llevaron al partido hegemónico a liberalizar las condiciones políticas para permitir cierto grado de competencia electoral, limitada y controlada por el régimen. ${ }^{11}$ Más tarde, los cambios en el ámbito interno e internacional generaron condiciones propicias para la transición

9 Medina Torres, Luis Eduardo y Ramírez Díaz, Edwin Cuitláhuac, op. cit., pp. 33-46.

10 Ibidem, pp. 42 y ss.

11 Por ejemplo, el trabajo ya clásico: Salazar, Pedro et al., La mecánica del cambio político en México: elecciones, partidos y reformas, México, Cal y Arena, 2000. 
democrática, que siguió siendo controlada por el régimen, y consistió, básicamente, en el establecimiento de reglas electorales adecuadas para crear condiciones de competencia, sistema plural de partidos, y un diseño institucional que garantizara certeza y limpieza de las elecciones.

De esta manera, con la reforma "definitiva" de 1996 se creó un modelo de gobernanza que, con ajustes menores, siguió vigente hasta la reforma de 2014. Bajo este modelo, existían en México "sistemas paralelos": el federal y los 32 sistemas locales. La gobernanza a nivel federal era regulada por la legislación federal, mientras que cada entidad federativa tenía sus propios códigos y leyes en la materia. Por supuesto, la Constitución federal establecía ciertas reglas comunes (por ejemplo, el sistema mixto para la integración de los legislativos), pero en general y en todos los ámbitos, los estados gozaban de una amplia libertad legislativa, por lo que no podemos hablar de una jerarquización, en aquella etapa, entre los sistemas federal y locales, más bien se trataba de mundos paralelos.

Los niveles de administración correspondían con los niveles de reglas: tanto a nivel federal, como en las entidades, existían órganos electorales con la misma estructura: un órgano administrativo encargado de la organización electoral (además de algunas otras tareas) y un órgano jurisdiccional, con la tarea de revisión jurisdiccional y resolución de conflictos electorales. La estructura de las autoridades administrativas era básicamente la misma: órganos autónomos e independientes, con patrimonio propio, sujetos a la regulación correspondiente a su ámbito de competencias. No existía relación jerárquica entre la autoridad federal y las locales, aunque sí colaboración (por medio de convenios para la realización conjunta de ciertas tareas o colaboración en asuntos como el padrón electoral). En cuanto a los órganos jurisdiccionales, en los estados existía una importante variedad: desde salas electorales incorporadas a los tribunales constitucionales o superiores de las entidades federativas, o tribunales incorporados a los poderes judiciales, hasta organismos autónomos. A nivel federal se creó un tribunal federal, incorporado al Poder Judicial de la Federación, el cual obtuvo competencia para conocer los asuntos relacionados tanto con las elecciones federales como en las locales (en calidad de un órgano revisor). De esta manera, a pesar de los paralelismos en la legislación y organización electoral, la Federación y las entidades compartían la revisión jurisdiccional. El control regional de derechos humanos ya era vigente, y es en esa época donde se desarrolla y cobra fuerza, mediante la revisión directa (como en el ya mencionado caso Castañeda) y a través del control de convencionalidad realizado por las autoridades jurisdiccionales mexicanas. 
Bajo el modelo de 1996, las postulaciones fueron competencia exclusiva de los partidos políticos. Los ciudadanos no podían postularse a ningún cargo electivo por derecho propio, y los intentos, tanto en las entidades federativas como de revisión ante la Corte Interamericana de Derechos Humanos (CIDH), no prosperaron. Las reglas de competencia electoral se fueron transformando a lo largo de los años y a través de las reformas, concentrándose en los aspectos financieros y de propaganda política.

Es importante señalar que la evolución electoral de México nos permite evidenciar la naturaleza cíclica de la gobernanza electoral. Como pudimos ver, el establecimiento del sistema electoral que existe en México es resultado de un largo proceso evolutivo, en el cual los partidos políticos diseñaban las reglas, las autoridades las aplicaban en el marco de un proceso electoral, y la evaluación del proceso de aplicación de las reglas nos llevaba a una nueva reforma electoral, es decir, de regreso al establecimiento de las reglas. Al inicio de esa evolución, que se convirtió en el proceso de transición a la democracia, el punto central fueron las reglas de acceso al juego electoral y al Poder Legislativo, así como el diseño de las instituciones electorales. Cuando esos elementos fueron instaurados y consolidados, a través del funcionamiento del Instituto Federal Electoral (IFE) y del Tribunal Federal Electoral — que más tarde se convirtieron en el Instituto Nacional Electoral (INE) y en el Tribunal Electoral del Poder Judicial de la Federación (TEPJF), respectivamente-, la atención se movió hacia los puntos más finos del sistema electoral: las condiciones de competencia, relacionadas principalmente con el financiamiento y acceso a los medios de comunicación. Cada reforma y cada conjunto de reglas fueron puestos en práctica durante uno o dos procesos electorales, analizados por parte de los actores políticos y "perfeccionados" en las reformas subsecuentes. Así, hemos tenido una serie de reformas que han respondido al llamado por la equidad en la contienda: 1990 (acceso a los medios), 1993 (aportaciones privadas, fiscalización de las finanzas y acceso a los medios de comunicación), 1996 (acceso a los medios de comunicación y financiamiento público), 2007 (fiscalización, acceso a radio y televisión y otros aspectos de la comunicación política) y 2014 (autoridades electorales, fiscalización, partidos, entre otros), que claramente evidencian el ciclo: creación de reglas, interpretación y aplicación de reglas, y de regreso a las modificaciones legislativas. De acuerdo con lo señalado 
por Ramírez, ${ }^{12}$ y Medina y Ramírez, ${ }^{13}$ también estuvo presente el fenómeno de revisión regional. Un ejemplo es el caso Castañeda, ${ }^{14}$ en el cual la CIDH reconoció la libertad de la que gozan los estados para configurar las vías de participación electoral, pero obligó al Estado mexicano a crear un mecanismo efectivo que garantizara el acceso a la justicia a los ciudadanos que pretendían desafiar las decisiones de las autoridades electorales. Por supuesto, la reforma de 2014 es otro ejemplo de esa dinámica. ${ }^{15}$

\section{Nuevo modelo de gobernanza}

La reforma de 2014 modificó las reglas electorales en México de manera sustancial, con lo que se estableció un nuevo modelo de gobernanza electoral. Los cambios trascendentales afectaron al diseño institucional, al modificar tanto la estructura como la distribución de las facultades de organización electoral. En primer lugar, la reforma desapareció al IFE y, en su lugar, creó al INE. La función básica del INE es la de organizar las elecciones federales, sin embargo, se le otorgaron también facultades en el ámbito local. En principio, es la autoridad supervisora y quien emite lineamientos para el desarrollo de los procesos locales. ${ }^{16}$ Adicionalmente, puede organizar las elecciones de los dirigentes de los partidos políticos, a petición de los mismos; ${ }^{17}$ tiene a su cargo la verificación del requisito necesario para realizar las consultas populares; también la organización, desarrollo, cómputo y declaración de resultados de las mismas (artículo 35, fracción VIII, numeral ocho), así como la tarea de la fiscalización de finanzas de los partidos, tanto en el ámbito federal, como local (artículo 41, base V, apartado B).

El Consejo General del INE obtuvo nuevas facultades, y puede, en los supuestos previstos por la ley y por el voto de al menos ocho consejeros:

12 Ramírez Díaz, Edwin Cuitláhuac, tesis doctoral “Gobernanza electoral en Centroamérica”, UAM-Iztapalapa, 27 de noviembre de 2013.

13 Medina Torres, Luis Eduardo y Ramírez Díaz, Edwin Cuitláhuac, op. cit., pp. 33-46.

14 Caso Castañeda Gutman vs. México, Corte Interamericana de Derechos Humanos, 30 de noviembre de 2007; disponible en $h t t p: / / w w w . c o r t e i d h . o r . c r / d o c s / r e s u m e n / c a s t a n e-$ da_gutman.pdf (fecha de consulta: 18 de marzo de 2016).

15 Gilas, Karolina M., Sistema de comunicación política a partir de la reforma 2014, México, TEPJF, en prensa.

16 Constitución Política de los Estados Unidos Mexicanos, artículo 41, base V, apartado $\mathrm{B}$, inciso a) y b).

17 Ibidem, artículo 41, base V, apartado B. 
- Asumir directamente la realización de las actividades propias de la función electoral que corresponden a los órganos electorales locales;

- Delegar en los órganos electorales locales las atribuciones relacionadas con la organización electoral, sin perjuicio de reasumir su ejercicio directo en cualquier momento;

- Atraer a su conocimiento cualquier asunto de la competencia de los órganos electorales locales, por su trascendencia o para sentar un criterio de interpretación (artículo 41, base V, apartado C).

Con la reforma, se incrementó el número de consejeros electorales a 11 y cambió el procedimiento de nombramiento del Consejo General. El nuevo procedimiento prevé una evaluación a cargo de un comité técnico, aunque el nombramiento se lleva a cabo por el voto de las dos terceras partes de los miembros presentes de la Cámara de Diputados. ${ }^{18}$ Además, se creó un Servicio Profesional Electoral Nacional, cuya regulación, que abarca las reglas de selección, ingreso, capacitación, profesionalización, promoción, evaluación, rotación, permanencia y disciplina de los servidores públicos de los órganos ejecutivos y técnicos tanto del INE, como de los organismos públicos locales, es desarrollada por los reglamentos que apruebe el Consejo General del INE. ${ }^{19}$

La reforma mantuvo la existencia de los institutos electorales locales, aunque con importantes cambios en sus facultades, integración y nombramiento. A partir de la reforma, todos los consejos generales de los institutos locales se integran por seis consejeros y un consejero presidente. Su designación y remoción corre a cargo del Consejo General del INE. ${ }^{20}$ Entre sus facultades está el realizar los escrutinios y cómputos, declarar su validez y otorgar constancias a los candidatos ganadores, realizar los procedimientos de consultas populares en el ámbito local; también están encargados de administrar el acceso a las prerrogativas de los candidatos y partidos políticos, realizar las tareas de educación cívica, preparación de la jornada electoral, impresión de documentos y la producción de materiales electorales y, finalmente, de resultados preliminares; encuestas o sondeos de opinión; observación electoral, y conteos rápidos; la mayoría de esas facultades las desarrollarán conforme a los lineamientos establecidos por el INE. ${ }^{21}$

18 Ibidem, artículo 41, base V, apartado A.

19 Ibidem, artículo 41, base V, apartado D.

20 Ibidem, artículo 41, base V, apartado C.

${ }^{21}$ Ibidem, artículo 41, base V, apartado C. 
Del lado de las autoridades jurisdiccionales, la reforma mantuvo todas las facultades del TEPJF y añadió una nueva: la de resolver los asuntos que el INE someta a su conocimiento para la imposición de sanciones por violaciones relacionadas con la propaganda política, electoral y gubernamental, realización de actos anticipados de campaña y acceso a los medios de comunicación (artículo 41, base III, apartado D y artículo 99, base IX). En cuando a los órganos jurisdiccionales locales, la reforma determinó que éstos deben ser órganos autónomos, y no pueden ser parte de los poderes judiciales de las entidades federativas, estableció su conformación por un número impar de magistrados, electos por las dos terceras partes de los miembros del Senado de la República, previa convocatoria que para tal efecto se emita (artículo 116, norma IV, inciso c, 5o).

Finalmente, respecto a las reglas de competencia electoral, la reforma reguló la postulación de ciudadanos por derecho propio (reconocida desde la reforma constitucional de 2012), unificó las reglas de registro de los partidos a nivel federal y estatal, modificó los esquemas de financiamiento público para los partidos políticos en las entidades federativas, aumentó la cantidad de recursos privados que pueden recibir los partidos, permitió a los medios de comunicación organizar los debates, además de introducir el principio de paridad de género en la postulación de candidatos.

Aplicando las categorías propuestas por Medina y Ramírez, ${ }^{22}$ podemos notar que el modelo construido por la reforma de 2014 se aleja notablemente de su antecesor. El nuevo esquema se basa en la centralización de la función electoral. Para lograrlo, la legislación electoral fue modificada, dejando atrás al Código Federal de Instituciones y Procedimientos Electorales que regulaba a las elecciones federales, y estuvo acompañada de las legislaciones locales. En su lugar, se adoptaron la Ley General de Instituciones y Procedimientos Electorales y la Ley General de Partidos Políticos, que regulan las elecciones, tanto en el ámbito federal como local. Si bien, las entidades federativas mantienen ciertos ámbitos de competencia, éstas quedan ya muy limitadas, pues la mayoría de las reglas electorales se vuelven uniformes a lo largo del país, lo que aplica para las elecciones federales y locales.

Aunque se mantuvo el esquema con dos niveles de administración electoral, ahora federal y local, sus ámbitos de competencia fueron modificados. En primer lugar, la autoridad nacional - INE - adquirió facultades relativas a la organización de los comicios locales, tanto de manera

22 Medina Torres, Luis Eduardo, Ramírez Díaz, Edwin Cuitláhuac, op. cit. 
directa (están a su cargo, por ejemplo, la geografía electoral, la ubicación e integración de las mesas directivas de casilla) como indirecta (emisión de lineamientos relativos a los conteos rápidos y el Programa de Resultados Electorales Preliminares, diseño e impresión de los materiales electorales, entre otros). En el segundo, el INE se convirtió en un superior jerárquico de los organismos públicos locales electorales (OPLE), a partir de varios elementos: i) le corresponde nombrar y remover a los consejeros locales; ii) puede atraer asuntos o asumir realización de algunas tareas e, incluso, de una elección local; iii) regula el ejercicio de la mayoría de las facultades de los OPLE, a través de lineamientos y reglamentos; iv) y controla al servicio profesional electoral a nivel local.

El tercer elemento es la uniformidad en la estructura y nombramiento de las autoridades electorales locales, que son ya de competencia nacional. Los OPLE, a partir de la reforma, se integran por el mismo número de consejeros electorales (siete), quienes son nombrados por el Consejo General del INE. Los tribunales locales, a su vez, se convirtieron en órganos autónomos, con 3 o 5 magistrados nombrados por el Senado de la República. De esta manera se crea un sistema nacional de elecciones, encabezado por el INE y la legislación general.

En cuanto a los actores políticos, el nuevo esquema de gobernanza permite postulaciones por los partidos y por el derecho propio de los ciudadanos, aunque no garantiza equidad en la contienda para ambos tipos de candidatos. Las demás reglas de competencia, relativas a financiamiento y acceso a los medios de comunicación, se vieron afectadas en medida muy menor.

\section{V. ¿Un modelo mejor?}

El nuevo modelo de gobernanza electoral fue probado en 2014, en las elecciones más grandes en la historia del país. La elección simultánea de los diputados federales y de las autoridades de diferentes niveles en las dieciséis entidades federativas fue la prueba de fuego para las nuevas reglas e instituciones. La pregunta obligada es: ¿en qué medida el nuevo modelo cumplió con las expectativas?

La homogeneización de las reglas electorales, bajo regulación constitucional más robusta y la emisión de las leyes generales parecían, en principio, un mecanismo que iba a lograr elecciones de una mejor calidad y, más importante, cierta uniformidad a lo largo del país. Sin embargo, ese objetivo no se logró, debido a los errores internos (del diseño legislativo) y externos (de actuación de las autoridades). Las deficiencias en el diseño 
legislativo se dieron a raíz de las negociaciones políticas que, en su inicio, apostaban por una nacionalización total de la función electoral, pero que a última hora determinaron mantener un sistema dual, aunque limitado. Ese hecho está evidenciado en la redacción de la reforma (a nivel constitucional y de ley general), que deja importantes lagunas legales, mantiene normas contradictorias y, en general, regula un sólo supuesto de elecciones concurrentes. Las autoridades jurisdiccionales, a su vez, han optado por tomar una ruta opuesta a la del legislador, pretendiendo proteger el "federalismo electoral", con lo que han renunciado a la posibilidad de una mayor centralización y uniformidad de las reglas electorales, al privilegiar en todo momento la "libertad de configuración legislativa" de las entidades federativas.

De esta manera, tenemos un sistema con alto nivel de regulación, pero con muchas lagunas legales. Con ello, creció el margen de la incertidumbre jurídica, ya que las lagunas, contradicciones, límites borrosos entre los ámbitos de competencia, etcétera, requieren de interpretación judicial. Su consecuencia secundaria es la complejidad del derecho electoral y su crecimiento exponencial. Como señalaba Schedler, hace tiempo:

el cuerpo de reglas formales relevantes se está expandiendo continuamente, ya que abarca también los reglamentos internos del IFE al igual que toda la jurisprudencia electoral, incluidos los fallos del Tribunal Electoral y los acuerdos del mismo Consejo General. En consecuencia, el derecho electoral se presenta cada [vez] más como una materia enigmática que revela sus secretos solamente a un puñado de especialistas iniciados (quienes luego enfrentan dificultades considerables en el momento en que tratan de traducir su razonamiento legal en un lenguaje comprensible para el público en general). ${ }^{23}$

Las leyes generales no regularon para los comicios locales algunos aspectos clave relacionados con la competencia electoral, con lo que se genera una desigualdad entre los actores participantes. Ese fenómeno es muy evidente en el caso de los candidatos independientes, quienes deben cumplir con los requisitos de registro distintos en cada entidad. De esa manera, tenemos entidades que exigen a los ciudadanos interesados juntar firmas de apoyo equivalentes al 1 o $2 \%$ de los inscritos en la lista nominal (a nivel federal, Jalisco, San Luis Potosí), mientras que algunas

23 Schedler, Andreas, "Incertidumbre institucional e inferencias de imparcialidad: el caso del Instituto Federal Electoral", Política y Gobierno, vol. VII, núm. 2, segundo semestre de 2000, pp. 383-421. 
otras requieren 4 o 5\%, o incluso más (Tabasco, Campeche, Yucatán). También otros requisitos distan bastante, como por ejemplo la prohibición de haber sido dirigente de algún partido político, algunos años antes de la postulación (desde un año en Michoacán, hasta tres años en Chihuahua o en el Estado de México). Con ello, resulta que es más fácil ejercer el derecho de ser votado como candidato independiente en algunas entidades que en otras. Por supuesto, los candidatos independientes están en una situación mucho más complicada frente a los postulados por los partidos políticos, lo que afecta sus posibilidades de obtener una victoria electoral.

En cuanto al diseño de las autoridades, el modelo vigente resulta ser altamente cuestionable. Si bien sigue abierta la discusión sobre las ventajas de un modelo nacional frente a uno descentralizado, el modelo adoptado en México queda en algún punto entre esos dos. Sin embargo, más que lo mejor de los dos mundos, construimos un híbrido complejo, enredado, con duplicidad de funciones y competencias mal definidas, que resulta ser más costoso, engorroso y difícil de operar.

Entre las problemáticas del nuevo diseño, podemos señalar que los mecanismos de coordinación entre la autoridad nacional y las locales no están previstos por la ley, por lo que el grado de cooperación depende, en gran medida, de la disposición de las personas que integran los órganos electorales. Ciertas funciones, como impresión de los materiales electorales, se encuentran en el ámbito de actuación de las autoridades de ambos niveles. El INE emite lineamientos en los que establece reglas para el diseño y elaboración de los materiales, mientras que los OPLE están a cargo de ejecutar, de manera directa, esa tarea. Dado que en la mayoría de las entidades federativas no existen empresas capaces de realizar la impresión conforme a los estándares de seguridad exigidos por la ley y por los lineamientos del INE, los OPLE deben gestionar esa tarea en la capital, en los mismos talleres en los que se imprimen las boletas para las elecciones federales. Ante el nuevo diseño, concentrador y unificador, hay que preguntarnos qué sentido tiene ese tipo de división de tareas, que vuelve su realización más complicada y más cara. ¿Si el INE determina el diseño de los materiales, no sería más sencillo, efectivo y barato que se encargará también de su impresión, conforme a las listas de candidatos proporcionados por los OPLE?

A partir de la reforma, la fiscalización de los partidos y candidatos en el ámbito federal y local es la facultad exclusiva del INE. Sin embargo, los OPLE mantienen todavía estructuras de fiscalización, ya que quedó bajo su competencia la revisión de los ingresos y gastos de las agrupacio- 
nes políticas. Además, el nuevo sistema de fiscalización que concentra esa tarea en el INE y lo obliga a fiscalizar todas las campañas electorales (federales y locales) en un plazo de apenas cuarenta días posteriores a la jornada comicial resulta ser ineficiente, al no tener la capacidad de detectar las irregularidades importantes. ${ }^{24}$

Finalmente, se esperaba que las elecciones, bajo el nuevo esquema, fueran más baratas a partir de la reforma. Sin embargo, el análisis del ejercicio electoral de 2015 no nos permite llegar a esa conclusión, debido a la ya descrita duplicidad de funciones y excesiva regulación en la materia. Como señala Ugalde, ${ }^{25}$ "el costo presupuestario de la democracia electoral en 2015 - tanto en el ámbito nacional como estatal- es de 34 mil 505 millones de pesos. En 2009, la última elección intermedia, fue de 26 mil 755 millones en pesos constantes, eso es un incremento en términos reales de $29 \%$ ".

\section{Conclusiones}

Desde la perspectiva analítica adoptada en ese trabajo, el nuevo modelo de gobernanza electoral resultó ser otro ejercicio fallido, al no atender los problemas más graves del sistema electoral mexicano. Se mantiene una legislación excesivamente regulada, engorrosa, confusa y contradictoria. La paradoja de la ley electoral mexicana -increíblemente detallada, pero, al mismo tiempo, llena de reglas imprecisas y contradictorias- sigue ahí.

Otro gran fenómeno - la judicialización de la política y de la función electoral- no sólo se mantiene, sino que crece a partir de la reforma. Las reglas requieren interpretación judicial, y las nuevas son objeto de pronunciamiento de las autoridades electorales con mayor frecuencia que en las épocas anteriores, en gran parte debido a la distribución de competencias establecida por la nueva legislación. En el TEPJF se ha notado la nueva dinámica, que significó un incremento de $147.88 \%$ respecto del número de asuntos recibidos en relación con los procesos electorales posteriores a la reforma $2007-2008 .{ }^{26}$ En ese contexto, la judicialización

24 Gilas, Karolina M. y Mikaela Kristin, Christiansson, "El nuevo modelo de fiscalización: La reforma fallida”, Revista Mexicana de Estudios Electorales, México, núm. 16, diciembre de 2015.

25 Ugalde, Luis Carlos, "Democracia a precio alzado", Nexos, 1 de agosto de 2015; disponible en $h t t p: / / w w w . n e x o s . c o m . m x / ? p=25810$ (fecha de consulta: 18 de marzo de 2016).

26 Informe Anual 2014-2015, TEPJF, 2015, p. 49; disponible en http://portal.te.gob.mx/ sites/default/files/Informe_2014_2015.pdf(fecha de consulta: 17 de marzo de 2016). 
suele reflejar cada vez mayor incertidumbre respecto del significado y alcances de las nuevas reglas.

También cabe señalar la inestabilidad del sistema electoral en México. Las reformas llegan con demasiada frecuencia; en los últimos veinte años hemos tenido cuatro reformas, en promedio cada cinco años, y desde 2015 se está hablando sobre la necesidad de aprobar una más. Ese ritmo de modificaciones legales genera una inestabilidad del sistema, supuestamente derivada de la necesidad de "perfeccionar" las reglas de juego. Sin embargo, los cambios que se aprueban no se basan en una evaluación profunda y un análisis racional de las problemáticas presentadas, sino que responden a las demandas de los perdedores de las elecciones, a las que los partidos ganadores ceden para conseguir su colaboración y legitimidad. Esa inestabilidad es una de las causas de la judicialización.

La reforma 2014 no va a ser definitiva, como tampoco lo fue la "gran reforma” de 1996. Como las demás reformas, ésta también fue reactiva, y se basó en una lista de quejas, más que en un análisis profundo de las fortalezas y debilidades del modelo anterior. Las deficiencias del modelo impiden romper con el ciclo de gobernanza y hacen suponer que, tarde o temprano (antes o después de la elección presidencial de 2018), se hagan otras modificaciones. La rueda electoral sigue girando. 